activity were assessed using mixed effects linear regression models in Stata 14.2.

Results $88.89 \%$ of children did not meet the recommended 180 min daily physical activity. Children spent a mean (SD) of 141.90 (33.10) minutes per day being physically active with 22.21 min per day $(S D=9.87)$ in MVPA. Children spent more minutes being active on nursery days than non-nursery days (146.89 vs $137.22, p=0.05)$. Boys were more physically active than girls, spending 148.95 vs $133.93 \mathrm{~min}$ in daily activity $(p=0.04)$. Older children were more physically active than younger children $(p=0.01)$. There were no differences in physical activity by parental education. Approximately half $(50.62 \%)$ of the sample spent less than $£ 9.00$ weekly on their 2-4-year-old's physical activity. Children scoring in the highest third of PedsQL physical functioning scores had higher levels of MVPA (4.06 95\% CI -0.41 to 8.54 , p-value 0.07). There was no evidence of a beneficial association between positive PedsQL psychosocial scores, or higher parental expenditure on physical activity, with more minutes spent being active or in MVPA.

Conclusion Physical activity was below the recommended $180 \mathrm{~min}$ of daily physical activity for this age group. Children were more physically active on nursery days. There is weak evidence of an association between better PedsQL physical scores and higher levels of MVPA. More time spent being physically active and in MVPA was not associated with higher expenditure on physical activity in this age group, but further examination in larger datasets is needed.

\section{OP79 THE EFFECT OF MOVING TO EAST VILLAGE (THE FORMER LONDON 2012 OLYMPIC GAMES ATHLETES VILLAGE) ON PHYSICAL ACTIVITY AND ADIPOSITY LEVELS}

${ }^{1} \mathrm{CM}$ Nightingale, ${ }^{1} \mathrm{~B}$ Ram, ${ }^{1} \mathrm{ES}$ Limb, ${ }^{1} \mathrm{~A}$ Shankar, ${ }^{2} \mathrm{D}$ Procter, ${ }^{2} \mathrm{AR}$ Cooper, ${ }^{2} \mathrm{AS}$ Page, ${ }^{3} \mathrm{C}$ Clary, ${ }^{3} \mathrm{D}$ Lewis, ${ }^{3} \mathrm{~S}$ Cummins, ${ }^{4} \mathrm{~A}$ Ellaway, ${ }^{5} \mathrm{~B}$ Giles-Corti, ${ }^{1} \mathrm{PH}$ Whincup, ${ }^{1} \mathrm{AR}$ Rudnicka, ${ }^{1} \mathrm{DG}$ Cook, ${ }^{1} \mathrm{CG}$ Owen*. ${ }^{1}$ Population Health Research Institute, St. George's, University of London, London, UK; ${ }^{2}$ Centre for Exercise, Nutrition and Health Sciences, University of Bristol, Bristol, UK; ${ }^{3}$ Department of Public Health, Environments and Society, London School of Hygiene and Tropical Medicine, London, UK; ${ }^{4} \mathrm{MRC} / \mathrm{SCO}$ Social and Public Health Sciences Unit, University of Glasgow, Glasgow, UK; ${ }^{5}$ Centre for Urban Research, RMIT University, Melbourne, Australia

\subsection{6/jech-2018-SSMabstracts.78}

Background There has been increasing interest in whether the built environmentinfluences health behaviours, but robust longitudinal evidence is limited. We assessed the effect of moving into East Village (the former London 2012 Olympic Games Athletes Village), a neighbourhood built on active design principles, on levels of physical activity (PA) and adiposity among adults.

Methods A cohort of 1278 adults $(16+)$ seeking to move into social, intermediate (a mixture of shared ownership, shared equity, affordable rent), and market-rent East Village accommodation were recruited in 2013-2015, and followed up after two years. Objective measures of PA using accelerometry (ActiGraph GT3X+), body mass index (BMI) and bioelectrical impedance (fat mass \%) were made. We examined change in levels of PA and adiposity, using multilevel models adjusting for sex, age group, ethnicity, housing sector (fixed effects) and household (random effect), comparing those who moved to East Village (intervention group) with those who lived outside
East Village (control group). Effects by housing sector and weekdays versus weekends for PA were also examined.

Results 877 adults (69\%) were followed-up, half had moved to East Village. Moving to East Village was associated with a small increase in daily steps $(151,95 \%$ CI $-233,534)$, more so in the intermediate sector $(399,95 \%$ CI $-211,1009)$ than in the social and market-rent sectors, but effects were not statistically significant. There were no differences in time spent in moderate-to-vigorous PA (MVPA) or any appreciable weekday versus weekend effects. There was no evidence of differences in time spent in light PA or sedentary time both overall and by housing sector with the exception of the market-rent group in whom moving to East Village was associated with a decrease in light PA ( -13.0 mins, 95\% CI $-24.7,-1.2$ mins). There were no effects of moving to East Village on BMI or fat mass $\%$ overall or by housing sector.

Conclusion At two-year follow-up, moving to East Village, a neighbourhood designed for healthy active living, did not have beneficial, consistent effects on objectively measured physical activity or adiposity of public health importance.

\section{OP80 EFFECTIVENESS OF EUROPEAN FANS IN TRAINING (EUROFIT): RANDOMISED CONTROLLED TRIAL IN ENGLAND, PORTUGAL, THE NETHERLANDS AND NORWAY}

${ }^{1} S$ Wyke ${ }^{*},{ }^{1} \mathrm{C}$ Bunn, ${ }^{2} \mathrm{E}$ Andersen, ${ }^{3} \mathrm{~J}$ Gill, ${ }^{1} \mathrm{CM}$ Gray, ${ }^{4} \mathrm{~K}$ Hunt, ${ }^{1} \mathrm{~A}$ McConnachie, ${ }^{5} \mathrm{~F}$ van Nassau, ${ }^{6} \mathrm{MN}$ Silva, ${ }^{5} \mathrm{HP}$ van der Ploeg. ${ }^{1}$ Institute of Health and Wellbeing, University of Glasgow, Glasgow, UK; ${ }^{2}$ Department of Coaching and Psychology, Norwegian School of Sport Science, Oslo, Norway; ${ }^{3}$ Institute of Cardiovascular and Medical Sciences, University of Glasgow, Glasgow, UK; ${ }^{4}$ Institute for Social Marketing, University of Stirling, Stirling, UK; ${ }^{5}$ Department of Public and Occupational Health, VU University Medical Centre, Amsterdam, The Netherlands; ${ }^{6}$ Interdisciplinary Center for the Study of Human Performance, University of Lisbon, Lisbon, Portugal

\subsection{6/jech-2018-SSMabstracts.79}

Background Despite widespread knowledge about the risks of unhealthy lifestyles, men can be reluctant to engage in lifestyle change programmes. Building on the success of the Scottish Football Fans in Training, we developed EuroFIT, a men-only, group-based lifestyle-change program to improve physical activity and reduce sitting time.

Our aim was to investigate whether EuroFIT can help men aged 30-65 years with a self-reported BMI $\geq 27 \mathrm{~kg} / \mathrm{m}^{2}$ to increase physical activity and decrease sitting time 12 months after baseline.

Methods We conducted a pragmatic, two-arm, randomised controlled trial in 15 football clubs in the Netherlands, Norway, Portugal and the UK (England). We measured participants at baseline, post-program and 12 months after baseline. Primary outcomes were objectively assessed changes in total physical activity (steps per day) and total sedentary time (minutes per day spent sitting). Secondary outcomes include self-reported food intake, weight, resting systolic and diastolic blood pressure and cardio-metabolic blood biomarkers. We conducted linear mixed effects regression analyses, including random effects for country and football club, and fixed effects for study group and baseline measurement.

Results 560 men were allocated to the intervention and objective measurements were achieved for 451 (81\%) at 12 months; 553 were allocated to the control and objective measurements were achieved for 470 (83\%). At 12 months, the mean difference in step count, adjusted for baseline steps and 
club, was 678 steps $(308,1048)$ in favour of the intervention $(\mathrm{p}<0.001)$; the mean difference in time spent sitting was $1.7 \mathrm{~min}(-10.4,13.8) \quad(p=0.78)$. We also found significicant improvements in self-reported food intake, weight, resting blood pressure and some cardio-metabolic blood biomakers in favour of the intervention. Seven serious adverse events were reported. Of these, 5 were assessed as likely to be associated with EuroFIT participation.

Discussion Participation in EuroFIT led to modest improvement in physical activity but not sedentary time at 12 months. Public health messages to be more physically active and eat well are now widely understood but the 'sit less' message is newer and less is known about how to achieve it. In this context coaches and participants may have found it difficult to disentangle advice about sitting less from advice about being more physical activity. Differences in outcomes between FFIT and EuroFIT will be discussed.

Authorship is on behalf of the EuroFIT consortium.

\section{OP81 APPLYING HURDLE MODELS TO ESTIMATE SOCIOECONOMIC INEQUALITIES IN MODERATE-TO- VIGOROUS PHYSICAL ACTIVITY: ANALYSIS USING THE HEALTH SURVEYS FOR ENGLAND 2008 AND 2012}

S Scholes*, JS Mindell. Epidemiology and Public Health, University College London, London, UK

\subsection{6/jech-2018-SSMabstracts.80}

Background Guidelines recommend adults engage in at least $150 \mathrm{~min}$ of moderate-to-vigorous physical activity (MVPA) per week. It is unknown whether describing socioeconomic inequalities using the average amount of time persons spend in MVPA masks: (1) disparities in the proportion of persons that are active, or (2) disparities in the amount of time that persons who are active spend engaging in physical activity.

Methods Hurdle models are a new way of accommodating continuous physical activity data with: (1) an excessive amount of zeros (non-participation), and (2) a continuous positivelyskewed part (the amount of time active persons spend being active). Using the Health Surveys for England $(n=16,012$; HSE 2008; 2012), we applied hurdle models to estimate inequalities in these two separate parts of MVPA data, and assess changes over time. Analyses were sex-specific and adjusted for body mass index and smoking. Separate analyses were performed for overall MVPA and for five activity domains, including walking and sports/exercise.

Results are presented as Marginal Effects (ME) with 95\% Confidence Intervals (95\% CIs). The MEs represent absolute differences between the highest- and lowest-income groups in: (1) the percentage of participants who were active, and (2) the average hours-per-week (hpw) spent in MVPA conditional on participants being active (i.e. hpw being greater than zero). Results The proportion of participants who performed any activity was highest in the highest-income group. The $\mathrm{ME}$ for overall MPVA was 12.5 percentage points (pp) [95\% CI 10.3 to $14.7 \mathrm{pp}$ ] in men; $11.6 \mathrm{pp}$ [9.5-13.7 pp] in women. Similar patterns were found for walking [men: $19.8 \mathrm{pp}$ : 16.7-22.8 pp; women: $15.0 \mathrm{pp}$ : $12.4-17.6 \mathrm{pp}]$ and for sports/exercise [men: 20.0 pp: 16.9-23.2 pp; women: 23.1 pp: 20.4-25.9 pp].

Differences in the amount of time spent in overall MVPA (amongst those doing any) also favoured high-income participants [men: 3.5 hpw: 2.4-4.7 hpw; women: 3.3 hpw: 2.5-4.2 hpw]. High-income participants spent on average 1 hpw more doing sports/exercise [men: 0.9 hpw: 0.0-1.8 hpw; women: $1.2 \mathrm{hpw}$ : 0.7-1.7 hpw]. However, time spent walking (amongst those doing any) showed the opposite pattern in men being $1.9 \mathrm{hpw}$ lower for those in the highest-income group [ -2.8 to $-0.9 \mathrm{hpw}$. Patterns were similar in 2008 and 2012. Findings were robust to different model specifications (e.g. using two-part models).

Conclusion Inequalities in overall MVPA and in sports/exercise were pronounced for the hurdle of participation and for the amount of time spent being active. For walking among men, inequalities were sharpest for the hurdle of participation, highlighting the importance of interventions designed to increase walking among inactive low-income individuals. Our results will be updated when HSE 2016 data are available (spring 2018).

\section{Health ageing}

\section{OP82 \#POPULATION PRIORITIES FOR SUCCESSFUL AGEING: A RANDOMISED VIGNETTE EXPERIMENT}

${ }^{1}$ E Whitley*, ${ }^{2} \mathrm{M}$ Benzeval, ${ }^{1} \mathrm{~F}$ Popham. ${ }^{1} \mathrm{MRC} / \mathrm{CSO}$ Social and Public Health Sciences Unit, University of Glasgow, Glasgow, UK; ${ }^{2}$ Institute for Social and Economic Research, University of Essex, Colchester, UK

\subsection{6/jech-2018-SSMabstracts.81}

Background Worldwide population ageing has resulted in a growing interest in 'successful ageing' but there is no established consensus as to what this entails. Existing evidence is largely qualitative, confounded, and restricted to older ages. We aimed to provide robust, unconfounded estimates of the relative importance placed by the general population on six commonly-used dimensions of successful ageing (disease, disability, physical functioning, cognitive functioning, interpersonal engagement, and productive engagement).

Methods We conducted a randomised experiment in wave 9 of the Understanding Society Innovation Panel, a stratified, geographically clustered sample of 2545 men and women designed to be representative of the British population. A total of 2010 (79\%) respondents took part in the experiment and were presented with three vignettes, each describing a hypothetical 75 year old person with randomly determined favourable or unfavourable outcomes for each of the six dimensions. Respondents were asked how successfully the person described was ageing on a scale from 0 (not successfully) to 10 (very successfully).

Results The main outcome measure for each of the dimensions was the difference in mean scores comparing vignettes with favourable versus unfavourable attributes; as each dimension was presented in the same way, direct comparisons can be made between them to understand their relative importance. Scores were allocated to 5967 vignettes and those in which dimensions were favourable were allocated higher scores than those in which they were unfavourable. However, the relative importance given to each dimension varied. Across all participants, the largest differences were observed for cognitive function (difference $(95 \% \mathrm{CI}): 1.20(1.11,1.30)$ ) and disability $(1.18(1.08,1.27))$ and the smallest for disease $(0.73(0.64$, $0.82))$ and productive engagement $(0.58(0.49,0.66)$. Differences for physical functioning and interpersonal engagement 